\title{
On the post-common-envelope central star of the planetary nebula NGC 2346
}

\author{
Alex J. Brown, ${ }^{1,2 \star}$ David Jones, ${ }^{3,4} \dagger$ Henri M. J. Boffin ${ }^{5}$, and Hans Van Winckel ${ }^{6}$ \\ ${ }^{1}$ Department of Physics and Astronomy, University of Sheffield, Sheffield, S3 7RH, UK \\ ${ }^{2}$ Isaac Newton Group of Telescopes, Apartado de Correos 368, E-38700 Santa Cruz de La Palma, Spain \\ ${ }^{3}$ Instituto de Astrofísica de Canarias, E-38205 La Laguna, Tenerife, Spain \\ ${ }^{4}$ Departamento de Astrofísica, Universidad de La Laguna, E-38206 La Laguna, Tenerife, Spain \\ ${ }^{5}$ European Southern Observatory, Karl-Schwarzschild-Str 2, 85748 Garching, Germany \\ ${ }^{6}$ Instituut voor Sterrenkunde, KU Leuven, Celestijnenlaan 200D bus 2401, 3001 Leuven, Belgium
}

Accepted XXX. Received YYY; in original form ZZZ

\begin{abstract}
The common-envelope phase is one of the most poorly understood phases of (binary) stellar evolution, in spite of its importance in the formation of a wide-range of astrophysical phenomena ranging from cataclysmic variables to cosmologically important supernova type Ia, and even recently discovered gravitational wave producing black hole mergers. The central star of the planetary nebula NGC 2346 has long been held as one of the longest period post-common-envelope systems known with a published period of approximately 16 days, however the data presented were also consistent with much shorter periods of around 1 day (a more typical period among the known sample of post-common-envelope binary central stars). Here, using the modern high-stability, high-resolution spectrograph HERMES, we conclusively show the period to, indeed, be 16 days while also revising the surface gravity to a value typical of a sub-giant (rather than main-sequence) resulting in an intrinsic luminosity consistent with the recently published GAIA parallax distance. Intriguingly, the implied mass for the secondary $\left(\gtrsim 3.5 \mathrm{M}_{\odot}\right.$ ) makes it, to our knowledge, the most massive post-common-envelope secondary known, whilst also indicating that the primary may be a post-RGB star.
\end{abstract}

Key words: binaries: spectroscopic - planetary nebulae: individual: NGC 2346 techniques: radial velocities

\section{INTRODUCTION}

Central star binarity is now thought to be a key ingredient in understanding the formation and evolution of a large fraction of planetary nebulae (PNe; Jones \& Boffin 2017) - playing an important role in the observed morphologies (Hillwig et al. 2016), chemistry (Wesson et al. 2018), and perhaps even in the planetary nebula luminosity function (PNLF; Ciardullo et al. 2005; Davis et al. 2018). However, very little is known about the processes by which binary stars can produce a $\mathrm{PN}$ - particularly the common envelope (CE) phase (see e.g. the review of Ivanova et al. 2013). One particularly interesting puzzle is the observed period distribution of post-CE central stars which shows a strong propensity of periods of a few days or less (Jones \& Boffin 2017), while models of the $\mathrm{CE}$ phase generally predict many more

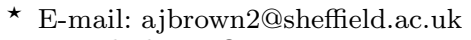

$\dagger$ E-mail: djones@iac.es
}

systems at longer periods (see section 4 of De Marco et al. 2008). As such, the properties of the few longer-period systems known, including recent discoveries by Manick et al. (2015), Sowicka et al. (2017) and Miszalski et al. (2018), are of particular interest - likely holding the key to resolving this disagreement and, perhaps, even to understanding the $\mathrm{CE}$ phase itself.

The binary central star of NGC 2346 was one of the first to be discovered (Méndez 1978), with a subsequent radial velocity study by Méndez \& Niemela (1981) deriving an orbital period of roughly 16 days making it one of the longest period post-CE binaries known to-date. However, there was considerable confusion over the true orbital period, with periods of around 1 day also presenting a reasonable fit to the data. Later photometric studies also found a dominant 16 day period but in this case not directly attributable to the orbital motion of the binary but rather due to (variable) occultations of the binary by a dust cloud (Mendez et al. 1982; Acker \& Jasniewicz 1985). Further support for such 
a long period comes from the observed nebular chemistry with Wesson et al. (2018) finding that PNe with shorter period binary central stars typically show extreme abundance discrepancies, while they place an upper limit on the abundance discrepancy factor of NGC 2346 of less than 10 (more consistent with a longer period central star).

As well as constraining the observed radial velocity variability, Méndez \& Niemela (1981) also derived the spectral type of the secondary star in the system (the hot primary is not visible in their optical spectra) concluding it to be an A-type star of mass $\mathrm{M}=1.8 \mathrm{M}_{\odot}$, temperature $\mathrm{T}_{\text {eff }}=8000 \mathrm{~K}$ and surface gravity $\log g=4.00$. These values imply a distance of $0.7 \pm 0.1 \mathrm{kpc}$ to the system, however the parallax as measured by GAIA result in a much larger distance of $1.45_{-0.08}^{+0.09} \mathrm{kpc}$ (Gaia Collaboration et al. 2018). This larger distance is also consistent with the distance to the nebula $(\mathrm{D}=1.57 \pm 0.54 \mathrm{kpc})$ as derived using the $\mathrm{H} \alpha$ surface brightness - radius relation of Frew et al. (2016).

In this paper, we present a study, based on VLT-FORS2 and Mercator-HERMES spectroscopy, of the central star of NGC 2346 to revisit the orbital period and stellar parameters in order to critically re-evaluate its status as one of the longest period post-CE binaries known as well as try to reconcile the apparent discrepancy between parallax distance and the distance implied by previous modelling attempts.

\section{RADIAL VELOCITY MONITORING}

The central star of NGC 2346 was observed 33 times (1800-s exposure time), between 2016 November 27 and 2018 April 18, using the Hermes Spectrograph mounted on the $1.2-\mathrm{m}$ Mercator Telescope at the Observatorio del Roque de Los Muchachos on the Spanish island of La Palma (Raskin et al. 2011). The pipeline reduced data were then continuum subtracted using the ISPEC software Blanco-Cuaresma et al. (2014) and cross-correlated against an A-type spectral template produced using SPECTRUM (Gray \& Corbally 1994). The resulting heliocentric radial velocity measurements are shown in table A1.

The radial velocities were then fit using the RADVEL package (Fulton et al. 2018), sampling the posterior probability densities of the orbital parameters (period, semiamplitude, eccentricity, argument of periastron) via Markov Chain Monte Carlo. The data are shown folded on the resulting best fit solution in figure 1 while the parameters of the fit and their uncertainties are listed in table 1. Fits were attempted forcing shorter orbital periods (with $\mathrm{P} \sim 1 \mathrm{~d}$ consistent with the possible periodicities identified by Méndez \& Niemela 1981), but in all cases the quality of the fit was significantly poorer than for a period of 16 days. In figure 2, we present a CLEANed power spectrum, computed via ten iterations with a loop gain of 0.1 (Roberts et al. 1987), of the radial velocity observations clearly showing that the $16 \mathrm{~d}$ period derived by the fit is the only convincing peak. As such, the new Mercator-HERMES data confirm the period favoured by Méndez \& Niemela (1981), fully ruling out the shorter period aliases which contaminated their data. We have also verified that adding the data from Méndez \& Niemela (1981) did not improve the orbit, given the very high quality data from Hermes.

The systemic velocity as measured by the fit

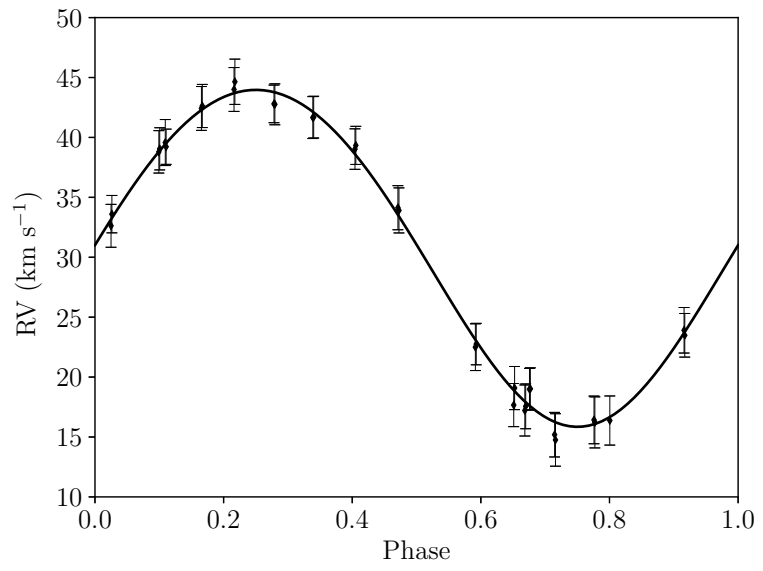

Figure 1. Radial velocity curve of NGC 2346

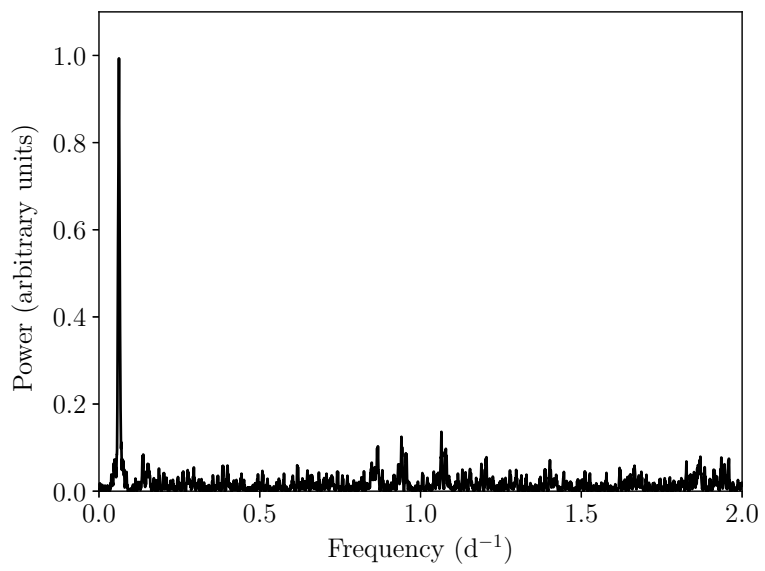

Figure 2. Power spectrum of the radial velocity observations of NGC 2346 showing the clear peak at a frequency of $0.0625 \mathrm{~d}^{-1}$ $(\mathrm{P}=16 \mathrm{~d})$.

$\left(\gamma=30.5 \pm 0.8 \mathrm{~km} \mathrm{~s}^{-1}\right)$ is appreciably different from the values quoted in the literature for the nebula (with most lying in the range 20-25 $\mathrm{km} \mathrm{s}^{-1}$; Durand et al. 1998; Méndez \& Niemela 1981). However, the systemic nebula velocity, as measured by fitting two gaussians (consistent with the two features arising from the front and back "walls" of the bipolar structure) to the [OIII] $5007 \AA$ emission line profile and taking the centroid (see figure 3 ), of our observations is found to be marginally consistent with the stellar systemic velocity $\left(v_{n e b}=32.7 \pm 1.6 \mathrm{~km} \mathrm{~s}^{-1}\right)$. Arias et al. (2001) provide support for a similar nebular velocity, as they find that the maximum intensity of their Fabry-Perot scans lies on the channel map at $27 \mathrm{~km} \mathrm{~s}^{-1}$, while of the channel maps either side of this the redder map (at $37 \mathrm{~km} \mathrm{~s}^{-1}$ ) is more symmetrical than its blue counterpart (at $17 \mathrm{~km} \mathrm{~s}^{-1}$ ) - indicative that the true systemic velocity lies between 27 and $37 \mathrm{~km} \mathrm{~s}^{-1}$ just as our measured values for both nebula and binary do. 
Table 1. Parameters of the central star of NGC 2346 derived from the Mercator radial velocities

\begin{tabular}{rl}
\hline Orbital period, $\mathrm{P}$ & $16.00 \pm 0.03 \mathrm{~d}$ \\
Systemic velocity, $\gamma$ & $30.5 \pm 0.8 \mathrm{~km} \mathrm{~s}^{-1}$ \\
Semi-amplitude, $\mathrm{K}$ & $14.1 \pm 0.6 \mathrm{~km} \mathrm{~s}^{-1}$ \\
Eccentricity, e & $0.04 \pm 0.03$ \\
Argument of periastron, $\omega$ & $180^{\circ} \pm 160^{\circ}$ \\
Binary mass function, $f$ & $0.00464 \pm 0.00062 \mathrm{M}_{\odot}$ \\
\hline Nebular systemic velocity & $32.7 \pm 0.6 \mathrm{~km} \mathrm{~s}^{-1}$ \\
\hline
\end{tabular}

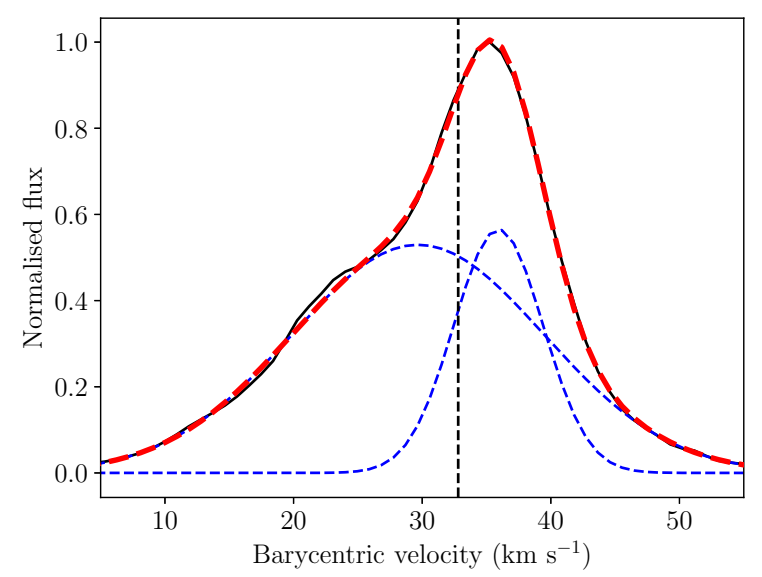

Figure 3. Gaussian fits of the [OIII] $5007 \AA$ emission from the PN NGC 2346.

\section{STELLAR PARAMETERS AND ABUNDANCES}

The central star of NGC 2346 was observed using the FORS2 instrument of the ESO VLT's UT1 Antu telescope (Appenzeller et al. 1998) with single spectra taken back-to-back using the 1200B (600-s exposure time) and 1200R (along with the GG435 order blocking filter, 60s exposure time) grisms. A $0.7^{\prime \prime}$ slit was employed with the MIT/LL CCD mosaic binned $2 \times 2\left(\equiv 0.25^{\prime \prime}\right.$ per binned pixel) to provide a spectral resolution of $1-2 \AA$ across the observed wavelength range $\lambda \sim 3600-5000 \AA$ (1200B grism) and $\lambda \sim 5800-7200 \AA$ (1200R grism). The spectra were biassubtracted, wavelength-calibrated and flux-calibrated using bias, arc lamp and standard star observations acquired as part of ESO's standard calibration plan. The spectra were then sky-subtracted (using only regions dominated by nebular emission, in order to subtract both sky and nebular contributions) before optimal extraction.

The temperature and surface gravity of the star were probed by comparing the resulting extracted spectra to synthetic spectra produced by SPECTRUM using model atmospheres from Castelli \& Kurucz (2004) which had been reddened assuming $\mathrm{E}(\mathrm{B}-\mathrm{V})=0.25$ and $\mathrm{R}_{v}=3.1$ (Frew et al. 2016). The best-fitting model (shown overlaid on the observed spectrum in figure 4) was found to have an effective temperature $T_{\text {eff }}=7750 \pm 200 \mathrm{~K}$ and surface gravity $\log$ $\mathrm{g}=3.0 \pm 0.25$. Consistent values were derived using the equivalent widths of iron lines present in the Mercator-Hermes spectrum presented in section 2. Furthermore, this analysis
Table 2. Atmospheric parameters for the SPECTRum model

\begin{tabular}{rll}
\hline Effective temperture, $\mathrm{T}_{\text {eff }}$ & 7750 & $\pm 200 \mathrm{~K}$ \\
Surface gravity, $\log (\mathrm{g})$ & 3.0 & $\pm 0.25 \mathrm{dex}$ \\
Metallicity, $[\mathrm{Fe} / \mathrm{H}]$ & -0.35 & $\pm 0.2 \mathrm{dex}$ \\
Microturbulence, $\xi$ & 4.0 & $\pm 0.1 \mathrm{~km} \mathrm{~s}^{-1}$ \\
Rotational velocity, vsini & 52 & $\pm 5 \mathrm{~km} \mathrm{~s}^{-1}$ \\
\hline
\end{tabular}

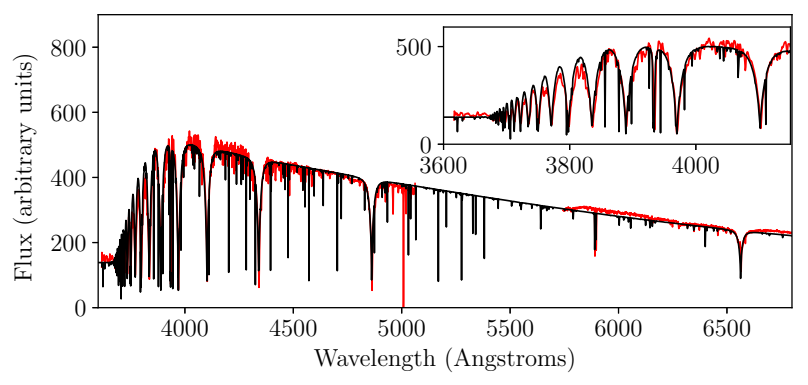

Figure 4. FORS2 spectrum of the central star of NGC 2346 (red) along with the best-fitting synthetic spectrum (black) implying an effective temperature, $\mathrm{T}_{\mathrm{eff}}=7750 \mathrm{~K}$, and a surface gravity, log $\mathrm{g}=3.0$.

allowed the derivation of the metallicity of the companion resulting in a best-fitting value for $[\mathrm{Fe} / \mathrm{H}] \sim-0.35$ (see figure 5). The quality of the fit is demonstrated for a selection of singly and doubly ionized Iron lines in figures 6. Lines of many other elements were present in the spectra including the s-process elements barium and strontium, and several elements from groups 2 (calcium, magnesium), 3 (scandium, yttrium) and 4 (titanium, zirconium). These were all probed for signs of inconsistency with respect to the derived metallicity (perhaps as a result of chemical contamination from the primary around the time of the $\mathrm{CE}$ phase, e.g. ; Miszalski et al. 2013), however no evidence for deviation in their abundances from those expected for the measured metallicity were found.

Collectively, the derived stellar parameters (listed in table Table 2) are consistent with evolutionary tracks of a sub-giant star of mass $\sim 3.5 \mathrm{M}_{\odot}$ (Bertelli et al. 2009). These tracks are extremely dependent on a multitude of factors, however a further sanity check of this mass is provided by the GAIA parallax distance $(\mathrm{D}=1.45 \mathrm{kpc})$. The luminosity of such a star $\left(\log (\mathrm{L} / \mathrm{L})_{\odot} \sim 2.4\right)$ implies an un-extincted apparent magnitude of approximately 10.8 at a distance of 1.45 kpc (assuming a typical bolometric correction of -0.2; Pickles 1998) - which is roughly consistent with the measured extinction corrected magnitude of Kohoutek (1995) at 10.9 mag, particularly when allowing for the effects of the observed large amplitude variability (Kohoutek 1983) and accounting for the uncertainties on the measured parameters (gravity, effective temperature, metallicity and extinction) and evolutionary tracks.

\section{DISCUSSION}

We have conclusively shown that the orbital period of the binary central star of NGC 2346 is, as previously derived 

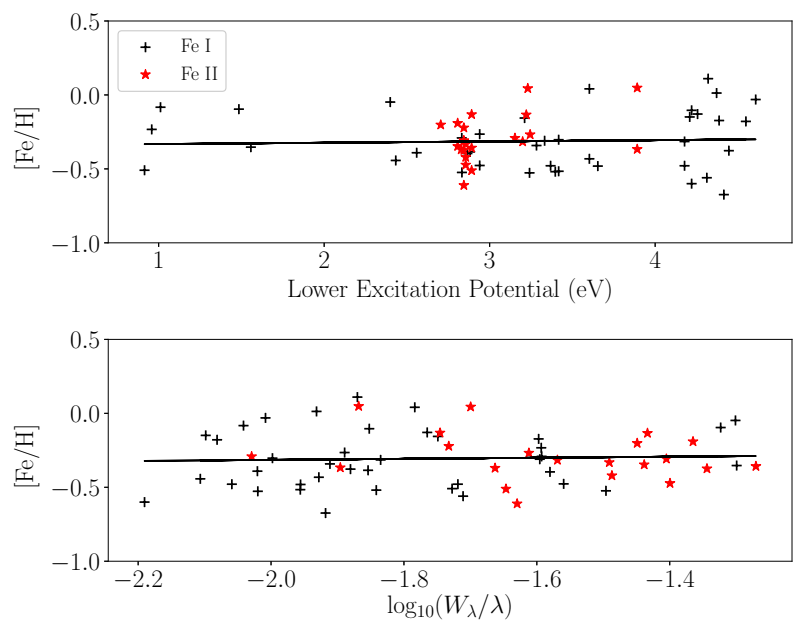

Figure 5. Iron abundances in the secondary component of the central star of NGC 2346 as a function of excitation potential (upper panel) and reduced equivalent width (lower panel).
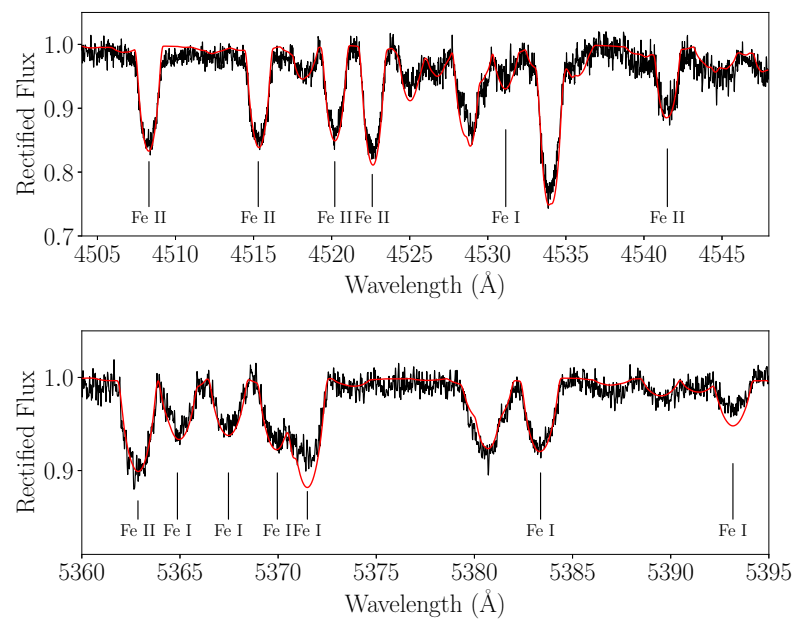

Figure 6. Observed (black) and synthetic (red) spectrum shown around two regions containing multiples lines of singly and doubly ionized iron, highlighting the quality of the fit. The synthetic spectrum was produced using parameters $\mathrm{T}_{\text {eff }}=7750 \mathrm{~K}, \log \mathrm{g}=3.0$ and $[\mathrm{Fe} / \mathrm{H}]=-0.35$

by Méndez \& Niemela (1981), approximately 16 days. Furthermore, detailed study of the stellar parameters via spectral synthesis revises the secondary in the system from a main-sequence star to a sub-giant, the absolute magnitude of which is consistent with the recently derived GAIA parallax distance.

At an inclination of $65^{\circ}$ (consistent with the binary plane being aligned with the waist of the bipolar nebula; Arias et al. 2001), and assuming a canonical mass of $0.6 \mathrm{M}_{\odot}$ for the primary, the binary mass function (derived from the Hermes radial velocity curve) implies a secondary mass of $\sim 5.3 \mathrm{M}_{\odot}$. For the derived surface gravity and temperature, such a massive companion would present with a luminosity inconsistent with the distance derived by GAIA. As such, either the binary plane is not aligned with the nebular waist (a lower inclination would imply a lower secondary mass), or the primary has a lower mass. The former option seems unlikely given that, in all cases where both are known, the binary orbital inclination is found to be coincident with the nebular waist - with a probability of chance a alignment being less than one in one million (Hillwig et al. 2016). The orbital period of NGC 2346 is significantly longer than the other systems, however all are post-CE meaning that one would not expect a misalignment ${ }^{1}$. It is, perhaps possible that the quoted inclination of the nebula is not accurate, with a value of $\sim 45^{\circ}$ bringing the secondary mass down to $3.5 \mathrm{M}_{\odot}$ (consistent with the derived atmospheric parameters and evolutionary tracks).

The second possibility, that the nebular progenitor has a lower mass, is perhaps equally unlikely. In order to be the more evolved component of the binary, the initial mass of the primary must have been greater than that of the secondary which, given that we do not see any evidence for chemical contamination in its atmosphere, is likely to be very similar to its current mass ${ }^{2}$. The white dwarf initial-final mass relation would imply that the primary's mass should be $\sim 0.8$ $\mathrm{M}_{\odot}$ (e.g.; El-Badry et al. 2018), unless its evolution was cut at a particularly early stage by the CE. For example, assuming a mass of $3.5 \mathrm{M}_{\odot}$ for the secondary and an orbital inclination of $65^{\circ}$, the implied primary mass is $0.46 \mathrm{M}_{\odot}$ consistent with the system experiencing the $\mathrm{CE}$ while the primary was on the RGB. Models of post-RGB evolution have demonstrated that such systems are capable of ionizing PNe (Hall et al. 2013), and a handful of other candidate (much shorter orbital period) post-RGB PNe have been discovered (Hillwig et al. 2017). If the nebula is the product of a $\mathrm{CE}$ on the RGB, then it would be expected to be more massive (as the envelope mass on the RGB is greater than on the AGB). This is seemingly borne out by estimates of the molecular mass of the nebula performed by Arias et al. (2001), who concluded that for a distance of $0.69 \mathrm{kpc}$ the total molecular mass of the nebula was $\sim 0.8 \mathrm{M}_{\odot}$ which when scaled for to the new distance of $1.45 \mathrm{kpc}$ implies an estimated molecular mass of approximately $3.5 \mathrm{M}_{\odot}$. It is important to highlight that this value is extremely dependent on the adopted pre-shock density, with more extreme values resulting in almost an order of magnitude difference in the estimated molecular mass (see section 3.5.2 of Arias et al. 2001 , for a full discussion) - nonetheless this estimate (derived using an intermediate value of the pre-shock density)

1 Some evidence for misalignment between binary and nebula has been found in LoTr 5 (Jones et al. 2017; Aller et al. 2018). However, the orbital orbital period of the central star of LoTr 5 is much longer ( $2700 \mathrm{~d})$, clearly differentiating its evolution from the others considered in that it has not experienced a CE phase. ${ }^{2}$ It is possible that the mass transfer could have occurred before the primary could become significantly chemically enriched (i.e. while it was still on the RGB), which would not result in chemical enrichment of the secondary. However, other post-CE systems, where significant accretion from primary to secondary has occurred, indicate that the majority of the mass transfer occurs very shortly before entering the CE phase (Miszalski et al. 2013; Jones et al. 2015) - meaning that if there was significant transfer of non-chemically enriched material while the primary was on the RGB it is also likely that the system experienced the $\mathrm{CE}$ while the primary was still on the RGB. 
serves to show that the PN may, indeed, be the product of a $\mathrm{CE}$ while the primary was on the RGB.

In summary, we confirm the central star of NGC 2346 to be one of the longest period post-CE systems known (see e.g. Rebassa-Mansergas et al. 2012; Sowicka et al. 2017; Miszalski et al. 2018). Furthermore, the secondary of the system is found to be sub-giant which, in spite of the larger uncertainties involved, must be one of, if not the most, massive post-CE secondaries known (Davis et al. 2010; Zorotovic et al. 2010). These properties make the central star of NGC 2346 an important system with which to study the $\mathrm{CE}$ and particularly the dependence of the $\mathrm{CE}$ efficiency on the parameters of the stellar components, extending the parameter space to longer periods and higher secondary masses (where, intriguingly, an anti-correlation between secondary mass and CE efficiency has been found; Davis et al. 2012). Unfortunately, the mass of the primary (another key ingredient in extending the parameter space of these studies) is unknown, however, assuming that the binary orbital plane is at least close to aligned with the waist of the bipolar nebula would imply a low primary mass perhaps consistent with a post-RGB object rather than post-AGB. Alternatively, NGC 2346 may be the first post-CE system in which the binary plane is not coincident with the nebular waist.

\section{ACKNOWLEDGEMENTS}

We thank the anonymous referee for their useful comments. This research was based on observations obtained with the HERMES spectrograph, which is supported by the Fund for Scientific Research of Flanders (FWO), Belgium, the Research Council of KU Leuven, Belgium, the Fonds National de la Recherche Scientifique (FNRS), Belgium, the Royal Observatory of Belgium, the Observatoire de Genève, Switzerland and the Thüringer Landessternwarte Tautenburg, Germany. The Mercator telescope is operated thanks to grant number G.0C31.13 of the FWO under the "Big Science" initiative of the Flemish government. H.V.W. acknowledges support from the Research Council of the KU Leuven under grant number C14/17/082. This paper is based on observations made with ESO Telescopes at the La Silla Paranal Observatory under programme IDs 093.D-0038 and 096.D-0080. This research has been supported by the Spanish Ministry of Economy and Competitiveness (MINECO) under the grant AYA2017-83383-P. The authors wish to thank all observers of the HERMES consortium institutes (KU Leuven, ULB, Royal Observatory, Belgium, and Sternwarte Tautenburg, Germany) who contributed to this monitoring programme.

\section{REFERENCES}

Acker A., Jasniewicz G., 1985, A\&A, 143, L1

Aller A., Lillo-Box J., Vučković M., Van Winckel H., Jones D., Montesinos B., Zorotovic M., Miranda L. F., 2018, MNRAS, 476,1140

Appenzeller I., et al., 1998, The Messenger, 94, 1

Arias L., Rosado M., Salas L., Cruz-González I., 2001, AJ, 122,3293

Bertelli G., Nasi E., Girardi L., Marigo P., 2009, A\&A, 508, 355
Blanco-Cuaresma S., Soubiran C., Heiter U., Jofré P., 2014, A\&A, 569, A111

Castelli F., Kurucz R. L., 2004, ArXiv Astrophysics e-prints, astro-ph/040587,

Ciardullo R., Sigurdsson S., Feldmeier J. J., Jacoby G. H., 2005, ApJ, 629, 499

Davis P. J., Kolb U., Willems B., 2010, MNRAS, 403, 179

Davis P. J., Kolb U., Knigge C., 2012, MNRAS, 419, 287

Davis B. D., Ciardullo R., Jacoby G. H., Feldmeier J. J., Indahl B. L., 2018, ApJ, 863, 189

De Marco O., Hillwig T. C., Smith A. J., 2008, AJ, 136, 323

Durand S., Acker A., Zijlstra A., 1998, A\&AS, 132, 13

El-Badry K., Rix H.-W., Weisz D. R., 2018, ApJL, 860, L17

Frew D. J., Parker Q. A., Bojičić I. S., 2016, MNRAS, 455, 1459

Fulton B. J., Petigura E. A., Blunt S., Sinukoff E., 2018, PASP, 130, 044504

Gaia Collaboration et al., 2018, A\&A, 616, A1

Gray R. O., Corbally C. J., 1994, AJ, 107, 742

Hall P. D., Tout C. A., Izzard R. G., Keller D., 2013, MNRAS, 435, 2048

Hillwig T. C., Jones D., De Marco O., Bond H. E., Margheim S., Frew D., 2016, ApJ, 832, 125

Hillwig T. C., Frew D. J., Reindl N., Rotter H., Webb A., Margheim S., 2017, AJ, 153, 24

Ivanova N., et al., 2013, A\&ARv, 21, 59

Jones D., Boffin H. M. J., 2017, Nature Astronomy, 1, 0117

Jones D., Boffin H. M. J., Rodríguez-Gil P., Wesson R., Corradi R. L. M., Miszalski B., Mohamed S., 2015, A\&A, 580, A19

Jones D., Van Winckel H., Aller A., Exter K., De Marco O., 2017, A\&A, 600, L9

Kohoutek L., 1983, MNRAS, 204, 93P

Kohoutek L., 1995, A\&AS, 113, 107

Manick R., Miszalski B., McBride V., 2015, MNRAS, 448, 1789

Méndez R. H., 1978, MNRAS, 185, 647

Méndez R. H., Niemela V. S., 1981, ApJ, 250, 240

Mendez R. H., Gathier R., Niemela V. S., 1982, A\&A, 116, L5

Miszalski B., Boffin H. M. J., Corradi R. L. M., 2013, MNRAS, 428, L39

Miszalski B., Manick R., Mikołajewska J., Van Winckel H., Iłkiewicz K., 2018, PASA, 35, e027

Pickles A. J., 1998, PASP, 110, 863

Raskin G., et al., 2011, A\&A, 526, A69

Rebassa-Mansergas A., et al., 2012, MNRAS, 423, 320

Roberts D. H., Lehar J., Dreher J. W., 1987, AJ, 93, 968

Sowicka P., Jones D., Corradi R. L. M., Wesson R., García-Rojas J., Santander-García M., Boffin H. M. J., Rodríguez-Gil P., 2017, MNRAS, 471, 3529

Wesson R., Jones D., García-Rojas J., Boffin H. M. J., Corradi R. L. M., 2018, MNRAS, 480, 4589

Zorotovic M., Schreiber M. R., Gänsicke B. T., Nebot GómezMorán A., 2010, A\&A, 520, A86+

\section{APPENDIX A: RADIAL VELOCITY MEASUREMENTS}

This paper has been typeset from a $\mathrm{TE}_{\mathrm{E}} \mathrm{X} / \mathrm{A} \mathrm{T} \mathrm{E} \mathrm{X}$ file prepared by the author. 
Table A1. Radial velocity measurements of the central star of NGC 2346

\begin{tabular}{lcl}
\hline Barycentric Julian date & $\begin{array}{c}\text { Barycentric radial velocity } \\
\left(\mathrm{km} \mathrm{s}^{-1}\right)\end{array}$ \\
\hline 2457720.75501 & 19.0 & \pm 1.7 \\
2457720.77642 & 19.0 & \pm 1.8 \\
2457727.69405 & 39.6 & \pm 1.9 \\
2457727.71547 & 39.2 & \pm 1.5 \\
2457736.63952 & 17.2 & \pm 2.1 \\
2457736.66094 & 17.6 & \pm 1.9 \\
2457788.60053 & 23.9 & \pm 1.9 \\
2457788.61847 & 23.5 & \pm 1.8 \\
2457792.59375 & 42.4 & \pm 1.8 \\
2457792.61517 & 42.6 & \pm 1.8 \\
2457801.38023 & 15.2 & \pm 1.9 \\
2457801.40164 & 14.8 & \pm 2.2 \\
2457802.36027 & 16.4 & \pm 2.0 \\
2457802.38168 & 16.2 & \pm 2.1 \\
2457806.34486 & 32.6 & \pm 1.8 \\
2457806.36627 & 33.6 & \pm 1.6 \\
2457807.53482 & 38.8 & \pm 1.8 \\
2457807.55623 & 39.0 & \pm 1.8 \\
2457810.39931 & 42.8 & \pm 1.6 \\
2457810.42072 & 42.8 & \pm 1.7 \\
2457812.41361 & 39.0 & \pm 1.7 \\
2457812.43502 & 39.3 & \pm 1.6 \\
2457813.48179 & 34.1 & \pm 1.8 \\
2457813.50789 & 33.9 & \pm 1.9 \\
2457815.40881 & 22.5 & \pm 1.9 \\
2457815.43022 & 22.8 & \pm 1.7 \\
2457816.36321 & 17.7 & \pm 1.8 \\
2457816.38462 & 19.1 & \pm 1.8 \\
2457857.40471 & 44.0 & \pm 1.8 \\
2457857.42612 & 44.6 & \pm 1.9 \\
2458106.75374 & 16.4 & \pm 2.0 \\
2458227.36508 & 41.7 & \pm 1.8 \\
2458227.38649 & 41.7 & \pm 1.7 \\
\hline & &
\end{tabular}

